\title{
The Eigenvalue Problem for Caputo Type Fractional Differential Equation with Riemann-Stieltjes Integral Boundary Conditions
}

\author{
Wenjie $\mathrm{Ma}^{1}$ and Yujun Cui $\mathbb{D}^{2}$ \\ ${ }^{1}$ Department of Applied Mathematics, Shandong University of Science and Technology, Qingdao 266590, China \\ ${ }^{2}$ State Key Laboratory of Mining Disaster Prevention and Control Co-Founded by Shandong Province and the Ministry of \\ Science and Technology, Shandong University of Science and Technology, Qingdao 266590, China
}

Correspondence should be addressed to Yujun Cui; cyj720201@163.com

Received 13 June 2018; Accepted 30 July 2018; Published 12 August 2018

Academic Editor: Liguang Wang

Copyright (C) 2018 Wenjie Ma and Yujun Cui. This is an open access article distributed under the Creative Commons Attribution License, which permits unrestricted use, distribution, and reproduction in any medium, provided the original work is properly cited.

In this paper, we investigate the eigenvalue problem for Caputo fractional differential equation with Riemann-Stieltjes integral boundary conditions ${ }^{c} D_{0+}^{\theta} p(y)+\mu f(t, p(y))=0, y \in[0,1], p(0)=p^{\prime \prime}(0)=0, p(1)=\int_{0}^{1} p(y) d A(y)$, where ${ }^{c} D_{0+}^{\theta}$ is Caputo fractional derivative, $\theta \in(2,3]$, and $f:[0,1] \times[0,+\infty) \longrightarrow[0,+\infty)$ is continuous. By using the Guo-Krasnoselskii's fixed point theorem on cone and the properties of the Green's function, some new results on the existence and nonexistence of positive solutions for the fractional differential equation are obtained.

\section{Introduction}

The experience of the last few years has fully borne out the fact that the integer order calculus is not as widely used as fractional order calculus in some fields such as chemistry, control theory, and signal processing. On the remarkable survey of Agarwal, Benchohra, and Hamani [1] it is pointed out that fractional differential equations constitute a fundamental tool in the modeling of some phenomena (see also [2-4]). The use of fractional order is more accurate for the description of phenomena, so the study of fractional differential equations becomes the mainstream with the help of techniques of nonlinear analysis. We refer the reader to [536] for recent results. For example, in [9], the author studied the following fractional differential equation:

$$
{ }^{c} D^{\alpha} x(y)+f(y, x(y))=0, \quad 0<y<1,
$$

with boundary conditions

$$
\begin{aligned}
& x^{\prime}(0)=x^{\prime \prime}(0)=0, \\
& x(1)=\mu \int_{0}^{1} x(s) d s,
\end{aligned}
$$

where $\alpha \in(2,3], \mu \in[0,1)$, and ${ }^{c} D^{\alpha}$ is the Caputo derivative. They solved the above problem by means of classical fixed point theorems.

In [5], the boundary value problem for the following nonlinear fractional differential equation was discussed:

$$
\begin{aligned}
D_{0+}^{\alpha} x(y)+a(y) f\left(y, x(y), x^{\prime \prime}(y)\right)=0, & \\
& 0<y<1,
\end{aligned}
$$

with boundary conditions

$$
x(0)=x^{\prime}(0)=x^{\prime \prime}(0)=x^{\prime \prime}(1)=0,
$$

where $D_{0+}^{\alpha}$ is the Riemann-Liouville differentiation, $\alpha \in$ $(3,4]$. By using a fixed point theorem, a new result of the existence of three positive solutions is obtained.

In [15], the authors investigated the following class of BVP:

$$
{ }^{c} D_{0+}^{q} x(y)=f\left(y, x(y),{ }^{c} D_{0+}^{\sigma} x(y)\right), \quad 0<y<1,
$$

with boundary conditions

$$
\begin{aligned}
& x(0)=x^{\prime \prime}(0)=0, \\
& x^{\prime}(1)=\alpha x^{\prime \prime}(1),
\end{aligned}
$$


where $q \in(2,3), \sigma \in(1,2), f:[0,1] \times R \times R \longrightarrow R$ is a given function, and ${ }^{c} D_{0+}^{q}$ denotes the Caputo differentiation. The author investigated this problem by using Banach contraction principle, Leray-Schauder nonlinear alternative, properties of the Green's function, and Guo-Krasnoselskii fixed point theorem on cone. Similar problems can be referred to in [25].

In this paper, we investigate the eigenvalue problem for Caputo fractional boundary value problem with RiemannStieltjes integral boundary conditions

$$
\begin{aligned}
{ }^{c} D_{0+}^{\theta} p(y)+\mu f(y, p(y)) & =0, \quad y \in[0,1], \\
p(0) & =p^{\prime \prime}(0)=0, \\
p(1) & =\int_{0}^{1} p(y) d A(y),
\end{aligned}
$$

where $\theta \in(2,3), f:[0,1] \times[0, \infty) \longrightarrow(0, \infty)$ is continuous, $\mu>0$ is a parameter, ${ }^{c} D_{0+}^{\theta}$ is the Caputo fractional derivative, and $A$ is a bounded variation function with positive measures with

$$
B=\int_{0}^{1} y d A(y)<1 .
$$

Our proof is based on the properties of the Green's function and the Guo-Krasnosel'skii fixed point theorem on cone.

\section{Preliminaries}

In order to solve problem (7), we provide the properties related to problem (7).

Definition 1 (see [3]). The Caputo's fractional derivative of order $\theta>0$ for a function $x \in C^{n}[0,+\infty)$ is defined as

$$
\begin{array}{r}
{ }^{c} D_{0+}^{\theta} x(y)=\frac{1}{\Gamma(n-\theta)} \int_{0}^{y}(y-s)^{n-\theta-1} x^{(n)}(s) d s, \\
n-1<\theta<n,
\end{array}
$$

where $n$ is the smallest integer greater than or equal to $\theta$.

Lemma 2 (see [3]). Let $\theta>0$. If we assume $x \in C(0,1) \cap$ $L(0,1)$, then the fractional differential equation

$$
{ }^{c} D_{0+}^{\theta} x(y)=0
$$

has the general solution $x(y)=C_{0}+C_{1} y+\cdots+C_{n-1} y^{n-1}$, $C_{k} \in R, k=0,1 \ldots, n-1$, where $n$ is the smallest integer greater than or equal to $\theta$.

Lemma 3 (see [3]). Given that $x \in C(0,1) \cap L(0,1)$ with a fractional derivative of order $\theta$ that belongs to $C(0,1) \cap L(0,1)$. Then

$$
\begin{aligned}
& I_{0+}^{\theta}{ }^{c} D_{0+}^{\theta} x(y)=x(y)+C_{0}+C_{1} y+\cdots+C_{n-1} y^{n-1}, \\
& \qquad \text { for } C_{k} \in R, k=0,1 \ldots, n-1,
\end{aligned}
$$

where $n$ is the smallest integer greater than or equal to $\theta$.

Firstly, we consider the following linear Caputo fractional differential equation:

$$
\begin{aligned}
{ }^{c} D_{0+}^{\theta} p(y)+\sigma(y) & =0, \quad y \in[0,1], \\
p(0) & =p^{\prime \prime}(0)=0, \\
p(1) & =\int_{0}^{1} p(y) d A(y) .
\end{aligned}
$$

Lemma 4. Let $\theta \in(2,3]$ and assume that $\sigma \in C[0,1]$. Then $p$ is the solution of the above boundary value problem (12), if and only if $p$ satisfies the following integral equation:

$$
p(y)=\int_{0}^{1} G(y, s) \sigma(s) d s,
$$

where

$$
G(y, s)=\frac{1}{\Gamma(\theta)} \begin{cases}\frac{y}{1-B}\left[(1-s)^{\theta-1}-\int_{s}^{1}(y-s)^{\theta-1} d A(y)\right]-(y-s)^{\theta-1}, & 0 \leq s \leq y \leq 1, \\ \frac{y}{1-B}\left[(1-s)^{\theta-1}-\int_{s}^{1}(y-s)^{\theta-1} d A(y)\right], & 0 \leq y \leq s \leq 1,\end{cases}
$$

and

$$
B=\int_{0}^{1} y d A(y)<1 .
$$

Proof. Applying the fractional integral of $\operatorname{order} \theta$ to both sides of (12) for $y \in[0,1]$, we get the following formula:

$$
I_{0+}^{\theta}{ }^{c} D_{0+}^{\theta} p(y)+I_{0+}^{\theta} \sigma(y)=0 .
$$

According to $p(0)=p^{\prime \prime}(0)=0$ and Lemma 3 , we obtain

$$
p(y)=c y-\frac{1}{\Gamma(\theta)} \int_{0}^{y}(y-s)^{\theta-1} \sigma(s) d s
$$

where $c \in R$. Since $p(1)=\int_{0}^{1} p(y) d A(y)$, we deduce that

$$
\begin{aligned}
c- & \frac{1}{\Gamma(\theta)} \int_{0}^{1}(1-s)^{\theta-1} \sigma(s) d s \\
& =\int_{0}^{1}\left(c y-\frac{1}{\Gamma(\theta)} \int_{0}^{y}(y-s)^{\theta-1} \sigma(s) d s\right) d A(y) .
\end{aligned}
$$


Journal of Function Spaces

3

Therefore,

$$
\begin{aligned}
c= & \frac{1}{\Gamma(\theta)(1-B)}\left[\int_{0}^{1}(1-s)^{\theta-1} \sigma(s) d s\right. \\
& \left.-\int_{0}^{1} \int_{0}^{y}(y-s)^{\theta-1} \sigma(s) d s d A(y)\right] .
\end{aligned}
$$

Substituting the above equality into (17), one has

$$
\begin{gathered}
p(y)=\frac{y}{\Gamma(\theta)(1-B)}\left[\int_{0}^{1}(1-s)^{\theta-1} \sigma(s) d s\right. \\
\left.-\int_{0}^{1} \int_{0}^{y}(y-s)^{\theta-1} \sigma(s) d s d A(y)\right]-\frac{1}{\Gamma(\theta)}
\end{gathered}
$$

$\cdot \int_{0}^{y}(y-s)^{\theta-1} \sigma(s) d s$

$$
\begin{aligned}
& =\frac{y}{\Gamma(\theta)(1-B)}\left[\int_{0}^{1}(1-s)^{\theta-1} \sigma(s) d s\right. \\
& \left.-\int_{0}^{1} \int_{s}^{1}(y-s)^{\theta-1} d A(y) \sigma(s) d s\right]-\frac{1}{\Gamma(\theta)}
\end{aligned}
$$

$$
\cdot \int_{0}^{y}(y-s)^{\theta-1} \sigma(s) d s
$$

and
The proof is completed.

Lemma 5. The Green's function $G(y, s)$ has the following properties:

(i) $\Gamma(\theta) G(y, s) \leq(1 /(1-B))(1-s)^{\theta-1}$, for $y, s \in[0,1]$;

(ii) $\Gamma(\theta) G(y, s) \geq N(1-s)^{\theta-1}$, for $y \in[1 / 4,3 / 4]$ and $s \in$ $[0,1]$, where

$$
\begin{gathered}
N=\min \left\{\frac{1-\int_{0}^{1} y^{\theta-1} d A(y)}{4(1-B)},\right. \\
\left.\min _{y \in[1 / 4,3 / 4]} y(1-y)^{\theta-2}\right\} .
\end{gathered}
$$

Proof. (i) Obviously, the inequality $\Gamma(\theta) G(y, s) \leq(1 /(1-$ $B)(1-s)^{\theta-1}$ holds from the representation of $G(y, s)$.

(ii) In view of $B=\int_{0}^{1} y d A(y)<1$ and $\theta \in(2,3)$, we have $1-\int_{s}^{1} y^{\theta-1} d A(y)>0$.

For $y \leq s$, we have

$$
\begin{aligned}
& \frac{y}{(1-B)}\left[(1-s)^{\theta-1}-\int_{s}^{1}(y-s)^{\theta-1} d A(y)\right] \\
& \geq \frac{y}{(1-B)}\left[(1-s)^{\theta-1}-\int_{s}^{1}(y-y s)^{\theta-1} d A(y)\right] \\
& \geq \frac{y}{(1-B)}(1-s)^{\theta-1}\left[1-\int_{s}^{1} y^{\theta-1} d A(y)\right] \\
& \geq \frac{y\left(1-\int_{0}^{1} y^{\theta-1} d A(y)\right)}{(1-B)}(1-s)^{\theta-1} .
\end{aligned}
$$

For $s \leq y$, we have

$$
\begin{aligned}
& \frac{y}{(1-B)}\left[(1-s)^{\theta-1}-\int_{s}^{1}(y-s)^{\theta-1} d A(y)\right]-(y \\
& -s)^{\theta-1} \geq \frac{y}{(1-B)}\left[(1-s)^{\theta-1}\right. \\
& \left.-\int_{s}^{1}(y-y s)^{\theta-1} d A(y)\right]-(y-y s)^{\theta-1} \\
& =\frac{y(1-B+B)}{(1-B)}(1-s)^{\theta-1}-\frac{y}{(1-B)} \\
& \cdot \int_{s}^{1}(y-y s)^{\theta-1} d A(y)-y^{\theta-1}(1-s)^{\theta-1} \\
& =y(1-s)^{\theta-1}+\frac{y \int_{0}^{1} y d A(y)}{(1-B)}(1-s)^{\theta-1} \\
& -\frac{y}{(1-B)} \int_{s}^{1}(y-y s)^{\theta-1} d A(y)-y^{\theta-1}(1-s)^{\theta-1} \\
& =(1-s)^{\theta-1}\left(y-y^{\theta-1}\right) \\
& +\frac{y}{(1-B)}\left((1-s)^{\theta-1} \int_{0}^{1} y d A(y)\right. \\
& \left.-\int_{s}^{1}(y-y s)^{\theta-1} d A(y)\right)=(1-s)^{\theta-1}\left(y-y^{\theta-1}\right) \\
& +\frac{y}{(1-B)}(1-s)^{\theta-1}\left(\int_{0}^{1} y d A(y)\right.
\end{aligned}
$$


4

Journal of Function Spaces

$$
\begin{aligned}
& \left.-\int_{0}^{1} y^{\theta-1} d A(y)\right)=(1-s)^{\theta-1}\left(y-y^{\theta-1}\right) \\
& +\frac{y}{(1-B)}(1-s)^{\theta-1}\left(\int_{0}^{1}\left(y-y^{\theta-1}\right) d A(y)\right) \geq(1 \\
& -s)^{\theta-1}\left(y-y^{\theta-1}\right)=(1-s)^{\theta-1} y\left(1-y^{\theta-2}\right) .
\end{aligned}
$$

Thus, the above two inequalities yield the inequality in (ii). The proof is completed.

Let $X=C[0,1],\|p\|=\max _{y \in[0,1]}|p(y)|$; then $(X,\|\cdot\|)$ is a Banach space. We define the cone $P \subset X$ by

$$
P=\left\{p \in X: p(y) \geq N(1-B)\|p\|, y \in\left[\frac{1}{4}, \frac{3}{4}\right]\right\} \text {. }
$$

Let $A_{\mu}: X \longrightarrow X$ be the operator defined as

$$
\left(A_{\mu} p\right)(y)=\mu \int_{0}^{1} G(y, s) f(s, p(s)) d s .
$$

Thus, the fixed point of the above integral equation is equivalent to the solution of the BVP (7).

Lemma 6. $A_{\mu}(P) \subset P$ and $A_{\mu}: P \longrightarrow P$ is a completely continuous operator, where $A_{\mu}$ is defined in (26).

Proof. By Lemma 5, for $\forall p \in P$, we have

$$
\begin{aligned}
& \left(A_{\mu} p\right)(y)=\mu \int_{0}^{1} G(y, s) f(s, p(s)) d s \\
& \geq \frac{\mu N}{\Gamma(\theta)} \int_{0}^{1}(1-s)^{\theta-1} f(s, p(s)) d s \\
& \geq \mu N(1-B) \max _{t \in[0,1]} \int_{0}^{1} G(t, s) f(s, p(s)) d s \\
& =N(1-B)\left\|A_{\mu} p\right\|, \quad y \in\left[\frac{1}{4}, \frac{3}{4}\right] .
\end{aligned}
$$

Hence we have $A_{\mu}(P) \subset P$. Let $\Omega \subset P$ be bounded. Then there exists a constant $M>0$ such that $\|p\| \leq M$ for $\forall p \in \Omega$. Let $L_{1}=\max _{y \in[0,1], p \in[0, M]}(\mu f(y, p)+1)$. Then

$$
\begin{aligned}
\left(A_{\mu} p\right)(y) \leq \frac{L_{1}}{\Gamma(\theta)(1-B)} \int_{0}^{1}(1-s)^{\theta-1} d s, & \\
& y \in[0,1] .
\end{aligned}
$$

Thus, $A_{\mu}(\Omega)$ is bounded. Put $p \in \Omega$ and $y_{1}, y_{2} \in[0,1]$. We deduce that

$$
\begin{aligned}
& \left|\left(A_{\mu} p\right)\left(y_{1}\right)-\left(A_{\mu} p\right)\left(y_{2}\right)\right|=\mu \mid \int_{0}^{1} G\left(y_{1}, s\right) \\
& \quad \cdot f(s, p(s)) d s-\int_{0}^{1} G\left(y_{2}, s\right) f(s, p(s)) d s \mid
\end{aligned}
$$

$=\mu \mid \int_{0}^{y_{1}}\left[\frac{y_{1}}{1-B}\left((1-s)^{\theta-1}-\int_{s}^{1}\left(y_{1}-s\right)^{\theta-1} d A(y)\right)\right.$

$\left.-\left(y_{1}-s\right)^{\theta-1}\right] f(s, p(s)) d s+\int_{y_{1}}^{y_{2}} \frac{y_{1}}{1-B}\left[(1-s)^{\theta-1}\right.$

$\left.-\int_{s}^{1}\left(y_{1}-s\right)^{\theta-1} d A(y)\right] f(s, p(s)) d s$

$+\int_{y_{2}}^{1} \frac{y_{1}}{1-B}\left[(1-s)^{\theta-1}-\int_{s}^{1}\left(y_{1}-s\right)^{\theta-1} d A(y)\right]$

$f(s, p(s)) d s$

$-\int_{0}^{y_{1}}\left[\frac{y_{2}}{1-B}\left((1-s)^{\theta-1}-\int_{s}^{1}\left(y_{2}-s\right)^{\theta-1} d A(y)\right)\right.$

$\left.-\left(y_{2}-s\right)^{\theta-1}\right] f(s, p(s)) d s$

$-\int_{y_{1}}^{y_{2}}\left[\frac{y_{2}}{1-B}\left((1-s)^{\theta-1}-\int_{s}^{1}\left(y_{2}-s\right)^{\theta-1} d A(y)\right)\right.$

$\left.-\left(y_{2}-s\right)^{\theta-1}\right] f(s, p(s)) d s-\int_{y_{2}}^{1} \frac{y_{2}}{1-B}\left[(1-s)^{\theta-1}\right.$

$\left.-\int_{s}^{1}\left(y_{2}-s\right)^{\theta-1} d A(y)\right] f(s, p(s)) d s \mid$

$=\mu \mid \int_{0}^{y_{1}}\left[(1-s)^{\theta-1} \frac{y_{1}-y_{2}}{1-B}\right.$

$-\int_{s}^{1}\left(\frac{y_{1}}{1-B}\left(y_{1}-s\right)^{\theta-1}-\frac{y_{2}}{1-B}\left(y_{2}-s\right)^{\theta-1}\right) d A(y)$

$\left.-\left(y_{1}-s\right)^{\theta-1}+\left(y_{2}-s\right)^{\theta-1}\right] f(s, p(s)) d s$

$+\int_{y_{1}}^{y_{2}}\left[(1-s)^{\theta-1} \frac{y_{1}-y_{2}}{1-B}\right.$

$-\int_{s}^{1}\left(\frac{y_{1}}{1-B}\left(y_{1}-s\right)^{\theta-1}-\frac{y_{2}}{1-B}\left(y_{2}-s\right)^{\theta-1}\right) d A(y)$

$\left.+\left(y_{2}-s\right)^{\theta-1}\right] f(s, p(s)) d s$

$+\int_{y_{2}}^{1}\left[(1-s)^{\theta-1} \frac{y_{1}-y_{2}}{1-B}\right.$

$\left.-\int_{s}^{1}\left(\frac{y_{1}}{1-B}\left(y_{1}-s\right)^{\theta-1}-\frac{y_{2}}{1-B}\left(y_{2}-s\right)^{\theta-1}\right) d A(y)\right]$

$f(s, p(s)) d s \mid \geq \frac{\left(y_{1}-y_{2}\right) L_{1}}{1-B} \int_{0}^{y_{1}}(1-s)^{\theta-1} d s$

$+L_{1} \int_{s}^{1} \int_{0}^{y_{1}}\left[\frac{y_{2}}{1-B}\left(y_{2}-s\right)^{\theta-1}\right.$

$\left.-\frac{y_{1}}{1-B}\left(y_{1}-s\right)^{\theta-1}\right] d s d A(y)$ 


$$
\begin{aligned}
& +L_{1} \int_{0}^{y_{1}}\left[\left(y_{2}-s\right)^{\theta-1}-\left(y_{1}-s\right)^{\theta-1}\right] d s \\
& +\frac{\left(y_{1}-y_{2}\right) L_{1}}{1-B} \int_{y_{1}}^{y_{2}}(1-s)^{\theta-1} d s \\
& +L_{1} \int_{s}^{1} \int_{y_{1}}^{y_{2}}\left[\frac{y_{2}}{1-B}\left(y_{2}-s\right)^{\theta-1}\right. \\
& \left.-\frac{y_{1}}{1-B}\left(y_{1}-s\right)^{\theta-1}\right] d s d A(y) \\
& +L_{1} \int_{y_{1}}^{y_{2}}\left(y_{2}-s\right)^{\theta-1} d s+\frac{\left(y_{1}-y_{2}\right) L_{1}}{1-B} \int_{y_{2}}^{1}(1 \\
& -s)^{\theta-1} d s+L_{1} \int_{s}^{1} \int_{y_{2}}^{1}\left[\frac{y_{2}}{1-B}\left(y_{2}-s\right)^{\theta-1}\right. \\
& \left.-\frac{y_{1}}{1-B}\left(y_{1}-s\right)^{\theta-1}\right] d s d A(y) \\
& =\frac{\left(y_{1}-y_{2}\right) L_{1}}{1-B} \int_{0}^{1}(1-s)^{\theta-1} d s \\
& +L_{1} \int_{s}^{1} \int_{0}^{1}\left[\frac{y_{2}}{1-B}\left(y_{2}-s\right)^{\theta-1}\right. \\
& \left.-\frac{y_{1}}{1-B}\left(y_{1}-s\right)^{\theta-1}\right] d s d A(y) \\
& +L_{1} \int_{y_{1}}^{y_{2}}\left(y_{2}-s\right)^{\theta-1} d s+L_{1} \int_{0}^{y_{1}}\left[\left(y_{2}-s\right)^{\theta-1}-\left(y_{1}\right.\right. \\
& \left.-s)^{\theta-1}\right] d s=\frac{\left(y_{1}-y_{2}\right) L_{1}}{(1-B) \theta} \\
& +\mathrm{E}_{1} \int_{s}^{1}\left[\frac{y_{1}\left(y_{1}-1\right)^{\theta}}{(1-B) \theta}-\frac{y_{2}\left(y_{2}-1\right)^{\theta}}{(1-B) \theta}\right] d A(y) \\
& +L_{1} \int_{s}^{1}\left[\frac{y_{2}^{\theta+1}}{(1-B) \theta}-\frac{y_{1}^{\theta+1}}{(1-B) \theta}\right] d A(y) \\
& +L_{1}\left(\frac{y_{2}^{\theta}}{\theta}-\frac{y_{1}^{\theta}}{\theta}\right) \text {. }
\end{aligned}
$$

Since $y, y(y-1)^{\theta}, y^{\theta+1}, y^{\theta}$ are uniformly continuous on $[0,1]$, $A_{\mu}(\Omega)$ is equicontinuous, by using Arzela-Ascoli's theorem, we can prove that $A_{\mu}: P \longrightarrow P$ is completely continuous. The proof is completed.

The following Guo-Krasnoselskii's fixed point theorem is used to prove the existence of positive solution of (7).

Theorem 7 (see [37]). Let $P$ be a cone of real Banach space $X$ and let $\Omega_{1}$ and $\Omega_{2}$ be two bounded open sets in $X$ such that $0 \in \Omega_{1} \in \bar{\Omega}_{1} \in \Omega_{2}$. Let operator $A: P \cap\left(\bar{\Omega}_{2} \backslash \Omega_{1}\right) \longrightarrow P$ be completely continuous operator. If one of the following two conditions holds:

(1) $\|A p\| \leq\|p\|$ for all $p \in P \cap \partial \Omega_{1},\|A p\| \geq\|p\|$ for all $p \in P \cap \partial \Omega_{2}$,
(2) $\|A p\| \geq\|p\|$ for all $p \in P \cap \partial \Omega_{1},\|A p\| \leq\|p\|$ for all $p \in P \cap \partial \Omega_{2}$,

then $A$ has at least one fixed point in $P \cap\left(\bar{\Omega}_{2} \backslash \Omega_{1}\right)$.

\section{Existence of Positive Solutions}

In this section, we investigate the existence of positive solutions for integral boundary value problems of fractional differential equation (7).

For convenience, we denote them by

$$
\begin{aligned}
h_{0} & =\lim _{u \longrightarrow 0^{+}} \sup _{y \in[0,1]} \frac{f(y, u)}{u}, \\
h_{\infty} & =\lim _{u \longrightarrow+\infty} \sup _{y \in[0,1]} \frac{f(y, u)}{u}, \\
h_{0}^{*} & =\lim _{u \longrightarrow 0^{+}} \inf _{y \in[1 / 4,3 / 4]} \frac{f(y, u)}{u}, \\
h_{\infty}^{*} & =\lim _{u \longrightarrow+\infty} \inf _{y \in[1 / 4,3 / 4]} \frac{f(y, u)}{u}, \\
C & =\frac{1}{\Gamma(\theta+1)(1-B)}, \\
E & =\frac{N^{2}(1-B)}{\Gamma(\theta)} \int_{1 / 4}^{3 / 4}(1-s)^{\theta-1} d s .
\end{aligned}
$$

Theorem 8. Suppose that $C h_{0}<E h_{\infty}^{*}$ holds; then for $\mu \epsilon$ $\left(1 / h_{\infty}^{*} E, 1 / C h_{0}\right)$, problem (7) has a positive solution. Here we impose $h_{0}^{-1}=+\infty$ if $h_{0}=0$ and $\left[h_{\infty}^{*}\right]^{-1}=0$ if $h_{\infty}^{*}=+\infty$.

Proof. Let $\mu \in\left(1 / h_{\infty}^{*} E, 1 / C h_{0}\right)$ and $\epsilon>0$ satisfy

$$
\frac{1}{\left(h_{\infty}^{*}-\epsilon\right) E} \leq \mu \leq \frac{1}{C\left(h_{0}+\varepsilon\right)} \text {. }
$$

According to the definition of $h_{0}$, we know that there exists a constant $E_{1}>0$ such that

$$
f(y, u) \leq\left(h_{0}+\varepsilon\right) u, \quad \text { for } u \in\left[0, E_{1}\right], y \in[0,1] .
$$

Put $\Omega_{1}=\left\{p \in P:\|p\|<E_{1}\right\}$. Let $p \in P \cap \partial \Omega_{1}$. We have $\|p\|=E_{1}$ and

$$
\begin{aligned}
& \left(A_{\mu} p\right)(y)=\mu \int_{0}^{1} G(y, s) f(s, p(s)) d s \\
& \quad \leq \frac{\mu}{\Gamma(\theta)(1-B)} \int_{0}^{1}(1-s)^{\theta-1}\left(h_{0}+\varepsilon\right) p(s) d s \\
& \quad \leq \frac{\mu\left(h_{0}+\varepsilon\right)}{\Gamma(\theta)(1-B)} \int_{0}^{1}(1-s)^{\theta-1} d s \cdot\|p\| \\
& \quad=\left(h_{0}+\varepsilon\right) \mu C \cdot\|p\| \leq\|p\|, \quad y \in[0,1] .
\end{aligned}
$$

Therefore, $\left\|A_{\mu} p\right\| \leq\|p\|$ for $p \in P \cap \partial \Omega_{1}$.

By the definition of $h_{\infty}^{*}$, we know that there exists $E_{2}>0$ such that

$$
f(y, u) \geq\left(h_{\infty}^{*}-\epsilon\right) u, \quad \text { for } u \geq E_{2} \text { and } y \in\left[\frac{1}{4}, \frac{3}{4}\right] .
$$


Let $E_{3}=\max \left\{2 E_{1}, E_{2} / N(1-B)\right\}, \Omega_{2}=\{p \in P:\|p\|<$ $\left.E_{3}\right\}$. Then for $p \in P \cap \partial \Omega_{2}$, by (25) we have

$$
\min _{y \in[1 / 4,3 / 4]} p(y) \geq N(1-B)\|p\| \geq E_{2},
$$

and thus

$$
\begin{aligned}
& \left(A_{\mu} p\right)(y)=\mu \int_{0}^{1} G(y, s) f(s, p(s)) d s \\
& \geq \mu \int_{1 / 4}^{3 / 4} G(y, s) f(s, p(s)) d s \\
& \geq \frac{\mu N}{\Gamma(\theta)} \int_{1 / 4}^{3 / 4}(1-s)^{\theta-1} f(s, p(s)) d s \\
& \geq \frac{\mu N}{\Gamma(\theta)} \int_{1 / 4}^{3 / 4}(1-s)^{\theta-1}\left(h_{\infty}^{*}-\epsilon\right) p(s) d s \\
& \geq \frac{\mu N^{2}\left(h_{\infty}^{*}-\epsilon\right)(1-B)}{\Gamma(\theta)} \int_{1 / 4}^{3 / 4}(1-s)^{\theta-1}\|p\| d s \\
& =\mu E\left(h_{\infty}^{*}-\epsilon\right) \cdot\|p\| \geq\|p\| .
\end{aligned}
$$

Therefore, $\left\|A_{\mu} p\right\| \geq\|p\|$ for $p \in P \cap \partial \Omega_{2}$.

By Theorem 7 , if $\mu \in\left(1 / h_{\infty}^{*} E, 1 / C h_{0}\right)$, we assert that $A_{\mu}$ has a fixed point in $P \cap\left(\overline{\Omega_{2}} \backslash \Omega_{1}\right)$ and therefore problem (7) has at least one positive solution. The proof is completed.

Theorem 9. Assume that $h_{\infty} C<E h_{0}^{*}$ holds. Then for $\mu \in\left(1 / E h_{0}^{*}, 1 / h_{\infty} C\right)$, the problem (7) has at least a positive solution. Here we impose $\left[h_{0}^{*}\right]^{-1}=0$ if $h_{0}^{*}=+\infty$ and $\left[h_{\infty}\right]^{-1}=$ $+\infty$ if $h_{\infty}=0$.

Proof. Let $\mu \in\left(1 / E h_{0}^{*}, 1 / h_{\infty} C\right)$ and $\epsilon>0$ such that

$$
\frac{1}{\left(h_{0}^{*}-\epsilon\right) E} \leq \mu \leq \frac{1}{C\left(h_{\infty}+\varepsilon\right)} .
$$

According to the definition of $h_{0}^{*}$, there exists a constant $E_{4}>$ 0 such that

$$
f(y, u) \geq\left(h_{0}^{*}-\epsilon\right) u
$$

$$
\text { for } u \in\left(0, E_{4}\right] \text { and } y \in\left[\frac{1}{4}, \frac{3}{4}\right] \text {. }
$$

Put $\Omega_{3}=\left\{p \in P:\|p\|<E_{4}\right\}$. Let $p \in P \cap \partial \Omega_{3}$; we have

$$
\begin{aligned}
& \left(A_{\mu} p\right)(y)=\mu \int_{0}^{1} G(y, s) f(s, p(s)) d s \\
& \geq \mu \int_{1 / 4}^{3 / 4} G(y, s) f(s, p(s)) d s \\
& \geq \frac{\mu N}{\Gamma(\theta)} \int_{1 / 4}^{3 / 4}(1-s)^{\theta-1} f(s, p(s)) d s \\
& \geq \frac{\mu N}{\Gamma(\theta)} \int_{1 / 4}^{3 / 4}(1-s)^{\theta-1}\left(h_{0}^{*}-\epsilon\right) p(s) d s
\end{aligned}
$$

$$
\begin{aligned}
& \geq \frac{\mu N^{2}\left(h_{0}^{*}-\epsilon\right)(1-B)}{\Gamma(\theta)} \int_{1 / 4}^{3 / 4}(1-s)^{\theta-1}\|p\| d s \\
& =\mu E\left(h_{0}^{*}-\epsilon\right) \cdot\|p\| \geq\|p\| .
\end{aligned}
$$

Therefore, $\left\|A_{\mu} p\right\| \geq\|p\|$ for $p \in P \cap \partial \Omega_{3}$.

It follows from the definition of $h_{\infty}$ that there exists a constant $E_{5}>0$ such that

$$
f(y, u) \leq\left(h_{\infty}+\frac{\varepsilon}{2}\right) u, \quad \text { for } u \geq E_{5} \text { and } y \in[0,1]
$$

This together with the continuity of $f$ implies that

$$
f(y, u) \leq\left(h_{\infty}+\frac{\varepsilon}{2}\right) u+M
$$

for $u \in R$ and $y \in[0,1]$

holds for some $M>0$.

Let $E_{6}=\max \left\{2 E_{4}, E_{5}, 2 M / \varepsilon\right\}, \Omega_{4}=\left\{p \in P:\|p\|<E_{6}\right\}$. For $\forall p \in P \cap \partial \Omega_{4}$, we conclude that

$$
\begin{aligned}
& \left(A_{\mu} p\right)(y)=\mu \int_{0}^{1} G(y, s) f(s, p(s)) d s \\
& \leq \frac{\mu}{\Gamma(\theta)(1-B)} \int_{0}^{1}(1-s)^{\theta-1}\left(h_{\infty}+\frac{\varepsilon}{2}\right)\|p\| d s \\
& \quad+\frac{M \mu}{\Gamma(\theta)(1-B)} \int_{0}^{1}(1-s)^{\theta-1} d s \\
& =\mu C\left(\left(h_{\infty}+\frac{\varepsilon}{2}\right)\|p\|+M\right) \leq \mu C\left(h_{\infty}+\varepsilon\right)\|p\| \\
& \leq\|p\| .
\end{aligned}
$$

Therefore, $\left\|A_{\mu} p\right\| \leq\|p\|$ for $\forall p \in P \cap \partial \Omega_{4}$.

By Theorem 7 , if $\mu \in\left(1 / E h_{0}^{*}, 1 / h_{\infty} C\right)$, we conclude that $A_{\mu}$ has a fixed point in $P \cap\left(\overline{\Omega_{4}} \backslash \Omega_{3}\right)$, and so problem (7) has one positive solution. The proof is completed.

\section{Nonexistence of Positive Solutions}

In this section, we present some sufficient conditions for nonexistence of positive solution to integral boundary value problems of fractional differential equation (7).

Theorem 10. If $h_{0}<+\infty$ and $h_{\infty}<+\infty$, then there exists $a \mu^{*}>0$ such that problem (7) has no positive solution for $\mu \in\left(0, \mu^{*}\right)$.

Proof. Since $h_{0}<+\infty, h_{\infty}<+\infty$, we have $f(y, u) \leq n_{1} u$ for $u \in\left[0, r_{1}\right]$, and $f(y, u) \leq n_{2} u$ for $u \in\left[r_{2},+\infty\right)$, where $n_{1}, n_{2}, r_{1}, r_{2}$ are positive numbers with $r_{1}<r_{2}$. Let $n=$ $\max \left\{n_{1}, n_{2}, \max _{r_{1} \leq u \leq r_{2}}(f(y, u) / u)\right\}$; then we have $f(y, u) \leq$ $n u$ for $u \in[0,+\infty)$. Suppose $p_{0}(y)$ is a positive solution of problem (7); then we are going to prove that this leads to a 
contradiction for $0<\mu<\mu^{*}:=1 / C n$. Since $\left(A_{\mu} p_{0}\right)(y)=$ $p_{0}(y)$, for $y \in[0,1]$, then

$$
\begin{aligned}
\left\|p_{0}\right\| & =\left\|A_{\mu} p_{0}\right\| \\
& \leq \frac{\mu}{\Gamma(\theta)(1-B)} \int_{0}^{1}(1-s)^{\theta-1} f\left(y, p_{0}(y)\right) d s \\
& \leq \mu C n\left\|p_{0}\right\|<\left\|p_{0}\right\|,
\end{aligned}
$$

which is a contradiction. Therefore this completes the proof.

Theorem 11. If $h_{0}^{*}>0, h_{\infty}^{*}>0, f(t, u)>0$ for $t \in[1 / 4,3 / 4]$ and $u>0$, then there exists $a \mu^{*}>0$ such that problem (7) has no positive solution for all $\mu>\mu^{*}$.

Proof. Since $h_{0}^{*}>+\infty, h_{\infty}^{*}>+\infty$, we have $f(y, u) \geq m_{1} u$ for $u \in\left[0, r_{1}\right]$, and $f(y, u) \geq m_{2} u$ for $u \in\left[r_{2},+\infty\right)$, where $m_{1}, m_{2}, r_{1}, r_{2}$ are positive numbers and $r_{1}<r_{2}$. Let $m=\min \left\{m_{1}, m_{2}, \min _{r_{1} \leq u \leq r_{2}}(f(y, u) / u)\right\}>0$; then we have $f(y, u) \geq m u$ for $u \in[0,+\infty)$. Suppose $p_{1}(y)$ is a positive solution of problem (7); then we are going to prove that this leads to a contradiction for $\mu>\mu^{*}:=1 / \operatorname{EmN}(1-B)$. Since $A_{\mu} p_{1}=p_{1}$, then

$$
\begin{aligned}
\left\|p_{1}\right\| & =\left\|A_{\mu} p_{1}\right\| \geq \frac{\mu N}{\Gamma(\theta)} \int_{1 / 4}^{3 / 4}(1-s)^{\theta-1} f(s, p(s)) d s \\
& \geq \frac{\mu m N}{\Gamma(\theta)} \int_{1 / 4}^{3 / 4}(1-s)^{\theta-1} p(s) d s \\
& \geq \frac{\mu m N}{\Gamma(\theta)} \int_{1 / 4}^{3 / 4}(1-s)^{\theta-1} N(1-B)\left\|p_{1}\right\| d s \\
& \geq \mu \operatorname{EmN}(1-B)\left\|p_{1}\right\|>\left\|p_{1}\right\|,
\end{aligned}
$$

which is a contradiction. Therefore this completes the proof.

\section{Example}

Example 1. We consider the following fractional equation:

$$
\begin{aligned}
& { }^{c} D_{0+}^{5 / 2} p(y)+\mu\left(y p^{3}(y)-p(y)+e^{p(y)}-1\right)=0, \\
& y \in[0,1], \\
& p(0)=p^{\prime \prime}(0)=0, \\
& p(1)=\frac{1}{2} \int_{0}^{1} p(y) d y,
\end{aligned}
$$

where $\theta=5 / 2, f(y, p)=y p^{3}-p+e^{p}-1, A(y)=(1 / 2) y$, $B=1 / 4<1$. We obtain $C=8 / 15 \Gamma(5 / 2), N=3 / 64, E=$ $27(9 \sqrt{3}-1) / 1310720 \Gamma(5 / 2)$.

It is easy to see that, for all $p>0$,

$$
\sup _{y \in[0,1]} \frac{f(y, p)}{p}=p^{2}-1+\frac{e^{p}-1}{p}
$$

and

$$
\inf _{y \in[1 / 4,3 / 4]} \frac{f(y, p)}{p}=\frac{1}{4} p^{2}-1+\frac{e^{p}-1}{p} .
$$

Then $h_{0}=0, h_{\infty}^{*}=\infty$; from Theorem 8 , problem (45) has a positive solution.

Example 2. We consider the following fractional equation:

$$
\begin{aligned}
& { }^{c} D_{0+}^{5 / 2} p(y)+\mu(\sqrt[3]{p(y)}+y \ln (p(y)+3))=0, \\
& p(0)=p^{\prime \prime}(0)=0, \\
& \left.p(1)=\frac{1}{2} \int_{0}^{1} p(y) d y, 1\right],
\end{aligned}
$$

where $\theta=5 / 2, f(y, p)=\sqrt[3]{p}+y \ln (p+3), A(y)=(1 / 2) y$, and $B=1 / 4<1$. We obtain $C=8 / 15 \Gamma(5 / 2), N=3 / 64$, and $E=27(9 \sqrt{3}-1) / 1310720 \Gamma(5 / 2)$.

One can easily see that, for all $p>0$,

$$
\sup _{y \in[0,1]} \frac{f(y, p)}{p}=\frac{\sqrt[3]{p}+\ln (p+3)}{p}
$$

and

$$
\inf _{y \in[1 / 4,3 / 4]} \frac{f(y, p)}{p}=\frac{\sqrt[3]{p}+(1 / 4) \ln (p+3)}{p} .
$$

Then $h_{\infty}=0, h_{0}^{*}=\infty$; from Theorem 9, problem (48) has a positive solution.

Example 3. We consider the following fractional equation:

$$
\begin{aligned}
{ }^{c} D_{0+}^{5 / 2} p(y)+\mu y p(y) & =0, \quad y \in[0,1], \\
p(0) & =p^{\prime \prime}(0)=0, \\
p(1) & =\frac{1}{2} \int_{0}^{1} p(y) d y,
\end{aligned}
$$

where $\theta=5 / 2, f(y, p)=y p, A(y)=(1 / 2) y$, and $B=1 / 4<$ 1. We obtain $C=8 / 15 \Gamma(5 / 2), N=3 / 64$, and $E=27(9 \sqrt{3}-$ 1) $/ 1310720 \Gamma(5 / 2)$.

By direct calculation, we obtain that

$$
h_{0}=\lim _{p \rightarrow 0^{+}} \sup _{y \in[0,1]} \frac{f(y, p)}{p}=1<+\infty
$$

and

$$
h_{\infty}=\lim _{p \longrightarrow+\infty} \sup _{y \in[0,1]} \frac{f(y, p)}{p}=1<+\infty .
$$

Take $n=1$ and $\mu^{*}=45 \sqrt{\pi} / 32>0$. By Theorem 10, problem (51) has no positive solution for $0<\mu<\mu^{*}$. 
Example 4. We consider the following fractional equation:

$$
\begin{aligned}
{ }^{c} D_{0+}^{5 / 2} p(y)+\mu y p(y) & =0, \quad y \in[0,1], \\
p(0) & =p^{\prime \prime}(0)=0, \\
p(1) & =\frac{1}{2} \int_{0}^{1} p(y) d y,
\end{aligned}
$$

where $\theta=5 / 2, f(y, p)=y p, A(y)=(1 / 2) y$, and $B=1 / 4<$ 1. We obtain $C=8 / 15 \Gamma(5 / 2), N=3 / 64$, and $E=27(9 \sqrt{3}-$ 1) $/ 1310720 \Gamma(5 / 2)$.

Obviously, we can infer that

$$
h_{0}^{*}=\lim _{p \rightarrow 0^{+}} \inf _{y \in[1 / 4,3 / 4]} \frac{f(y, p)}{p}=\frac{1}{4}>0
$$

and

$$
h_{\infty}^{*}=\lim _{p \rightarrow+\infty} \inf _{y \in[1 / 4,3 / 4]} \frac{f(y, p)}{p}=\frac{1}{4}>0 .
$$

Take $m=1 / 4$ and $\mu^{*}=3.4 \sqrt{\pi} / 81(9 \sqrt{3}-1) \times 10^{8}$. By Theorem 11, problem (54) has no positive solution for $\mu>\mu^{*}$.

\section{Data Availability}

The dataset supporting the conclusions of this article is included within the article.

\section{Conflicts of Interest}

The authors declare that they have no conflicts of interest.

\section{Acknowledgments}

The research is supported by the National Natural Science Foundation (NNSF) of China (11371221, 11571207), Shandong Natural Science Foundation (ZR2018MA011), and the Tai'shan Scholar Engineering Construction Fund of Shandong Province of China.

\section{References}

[1] R. P. Agarwal, M. Benchohra, and S. Hamani, "A survey on existence results for boundary value problems of nonlinear fractional differential equations and inclusions," Acta Applicandae Mathematicae, vol. 109, no. 3, pp. 973-1033, 2010.

[2] R. Hilfer, Applications of Fractional Calculus in Physics, World Scientific, Singapore, 2000.

[3] A. A. Kilbas, H. M. Srivastava, and J. J. Trujillo, Theory and Applications of Fractional Differential Equations, Elsevier, New York, NY, USA, 2006.

[4] H. W. Yang, X. Chen, M. Guo, and Y. D. Chen, "A new ZK-BO equation for three-dimensional algebraic Rossby solitary waves and its solution as well as fission property," Nonlinear Dynamics, vol. 91, pp. 2019-2032, 2018.

[5] C. Bai, "Triple positive solutions for a boundary value problem of nonlinear fractional differential equation," Electronic Journal of Qualitative Theory of Differential Equations, vol. 2008, no. 16, pp. 1-10, 2008.
[6] Z. Bai, "On positive solutions of a nonlocal fractional boundary value problem," Nonlinear Analysis: Theory, Methods \& Applications, vol. 72, no. 2, pp. 916-924, 2010.

[7] Z. Bai, S. Zhang, S. Sun, and C. Yin, "Monotone iterative method for fractional differential equations," Electronic Journal of Differential Equations, vol. 2016, no. 6, pp. 1-8, 2016.

[8] M. Benchohra, A. Cabada, and D. Seba, "An existence result for nonlinear fractional differential equations on Banach spaces," Boundary Value Problems, Article ID 628916, 11 pages, 2009.

[9] A. Cabada, S. Dimitrijevic, T. Tomovic, and S. Aleksic, "The existence of a positive solution for nonlinear fractional differential equations with integral boundary value conditions," Mathematical Methods in the Applied Sciences, vol. 40, no. 6, pp. 1880-1891, 2017.

[10] A. Cabada and Z. Hamdi, "Nonlinear fractional differential equations with integral boundary value conditions," Applied Mathematics and Computation, vol. 228, pp. 251-257, 2014.

[11] Y. Cui, W. Ma, X. Wang, and X. Su, "Uniqueness theorem of differential system with coupled integral boundary conditions," Electronic Journal of Qualitative Theory of Differential Equations, vol. 2018, no. 9, pp. 1-10, 2018.

[12] Y. Cui, Q. Sun, and X. Su, "Monotone iterative technique for nonlinear boundary value problems of fractional order $p \epsilon$ $(2,3], "$ Advances in Difference Equations, vol. 2017, Article ID 248,2017

[13] Y. Cui, W. Ma, Q. Sun, and X. Su, "New uniqueness results for boundary value problem of fractional differential equation," Nonlinear Analysis: Modelling and Control, vol. 23, no. 1, pp. 3139, 2018.

[14] C. S. Goodrich, "Existence of a positive solution to a class of fractional differential equations," Applied Mathematics Letters, vol. 23, no. 9, pp. 1050-1055, 2010.

[15] A. Guezane-Lakoud and R. Khaldi, "Positive solution to a fractional boundary value problem," International Journal of Differential Equations, Article ID 763456, 19 pages, 2011.

[16] L. Guo, L. Liu, and Y. Wu, "Existence of positive solutions for singular fractional differential equations with infinite-point boundary conditions," Nonlinear Analysis, Modelling and Control, vol. 21, no. 5, pp. 635-650, 2016.

[17] X. Hao, H. Wang, L. Liu, and Y. Cui, "Positive solutions for a system of nonlinear fractional nonlocal boundary value problems with parameters and $p$-Laplacian operator," Boundary Value Problems, vol. 2017, Article ID 182, 2017.

[18] J. Jiang, L. Liu, and Y. Wu, "Positive solutions to singular fractional differential system with coupled boundary conditions," Communications in Nonlinear Science and Numerical Simulation, vol. 18, no. 11, pp. 3061-3074, 2013.

[19] J. Jiang, W. Liu, and H. Wang, "Positive solutions to singular Dirichlet-type boundary value problems of nonlinear fractional differential equations," Advances in Difference Equations, vol. 2018, Article ID 169, 2018.

[20] N. Kosmatov and W. Jiang, "Resonant functional problems of fractional order," Chaos, Solitons \& Fractals, vol. 91, pp. 573-579, 2016.

[21] L. Liu, X. Zhang, J. Jiang, and Y. Wu, "The unique solution of a class of sum mixed monotone operator equations and its application to fractional boundary value problems," The Journal of Nonlinear Science and Its Applications, vol. 9, no. 5, pp. 29432958, 2016.

[22] X. Lv, X. Meng, and X. Wang, "Extinction and stationary distribution of an impulsive stochastic chemostat model with 
nonlinear perturbation," Chaos, Solitons \& Fractals, vol. 110, pp. 273-279, 2018.

[23] T. Qi, Y. Liu, and Y. Zou, "Existence result for a class of coupled fractional differential systems with integral boundary value conditions," Journal of Nonlinear Sciences and Applications, vol. 10, no. 7, pp. 4034-4045, 2017.

[24] T. Qi, Y. Liu, and Y. Cui, "Existence of solutions for a class of coupled fractional differential systems with nonlocal boundary conditions," Journal of Function Spaces, vol. 2017, Article ID 6703860, 9 pages, 2017.

[25] M. U. Rehman and R. A. Khan, "Existence and uniqueness of solutions for multi-point boundary value problems for fractional differential equations," Applied Mathematics Letters, vol. 23, no. 9, pp. 1038-1044, 2010.

[26] Q. Sun, H. Ji, and Y. Cui, "Positive solutions for boundary value problems of fractional differential equation with integral boundary conditions," Journal of Function Spaces, vol. 2018, Article ID 6461930, 6 pages, 2018.

[27] J. Wu, X. Zhang, L. Liu, Y. Wu, and Y. Cui, “The convergence analysis and error estimation for unique solution of a $\mathrm{p}$ Laplacian fractional differential equation with singular decreasing nonlinearity," Boundary Value Problems, vol. 2018, Article ID 82, 2018.

[28] X. Zhang, L. Liu, Y. Wu, and Y. Cui, "The existence and nonexistence of entire large solutions for a quasilinear Schrödinger elliptic system by dual approach," Journal of Mathematical Analysis and Applications, vol. 464, no. 2, pp. 1089-1106, 2018.

[29] X. Zhang, L. Liu, Y. Wu, and Y. Zou, "Existence and uniqueness of solutions for systems of fractional differential equations with Riemann-Stieltjes integral boundary condition," Advances in Difference Equations, vol. 2018, Article ID 204, 2018.

[30] X. Zhang, Y. Wu, and Y. Cui, "Existence and nonexistence of blow-up solutions for a Schrödinger equation involving a nonlinear operator," Applied Mathematics Letters, vol. 82, pp. 85-91, 2018.

[31] X. Zhang and Q. Zhong, “Triple positive solutions for nonlocal fractional differential equations with singularities both on time and space variables," Applied Mathematics Letters, vol. 80, pp. $12-19,2018$.

[32] Y. Zou and G. He, "A fixed point theorem for systems of nonlinear operator equations and applications to $(p 1, p 2)$ Laplacian system," Mediterranean Journal of Mathematics, vol. 15, Article ID 74, 2018.

[33] Y. Zou and G. He, "On the uniqueness of solutions for a class of fractional differential equations," Applied Mathematics Letters, vol. 74, pp. 68-73, 2017.

[34] Y. Zou and G. He, "The existence of solutions to integral boundary value problems of fractional differential equations at resonance," Journal of Function Spaces, vol. 2017, Article ID 2785937, 7 pages, 2017.

[35] Y. Zou, L. Liu, and Y. Cui, “The existence of solutions for fourpoint coupled boundary value problems of fractional differential equations at resonance," Abstract and Applied Analysis, vol. 2014, Article ID 314083, 8 pages, 2014.

[36] M. Zuo, X. Hao, L. Liu, and Y. Cui, "Existence results for impulsive fractional integro-differential equation of mixed type with constant coefficient and antiperiodic boundary conditions," Boundary Value Problems, vol. 2017, Article ID 161, 2017.

[37] D. J. Guo and V. Lakshmikantham, Nonlinear Problems in Abstract Cones, vol. 5 of Notes and Reports in Mathematics in Science and Engineering, Academic Press, New York, NY, USA, 1988. 


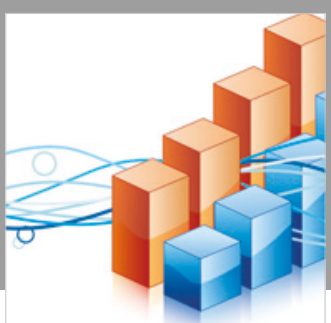

Advances in

Operations Research

\section{-n-m}
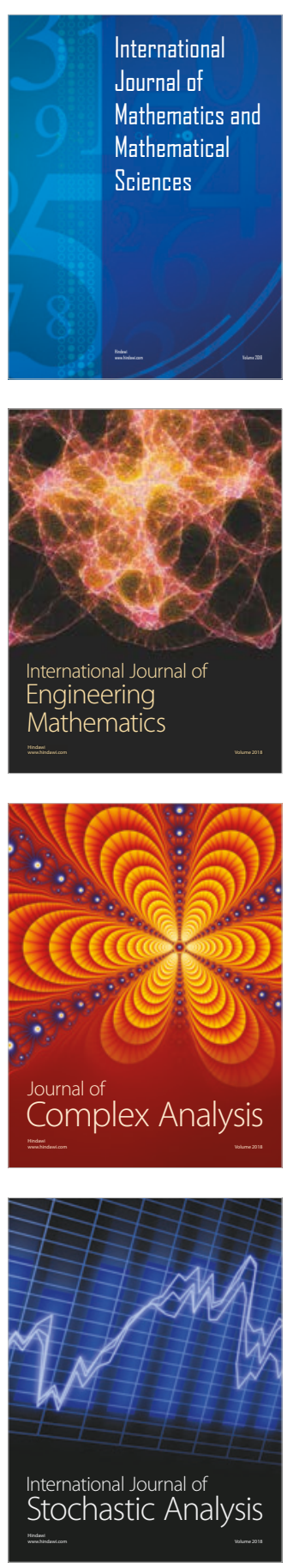
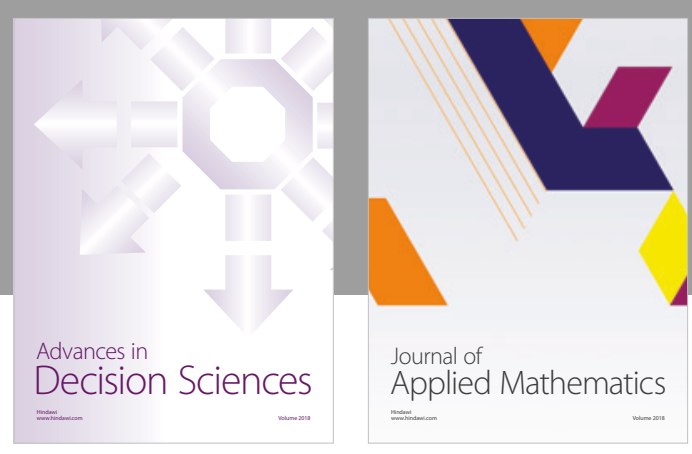

Journal of

Applied Mathematics
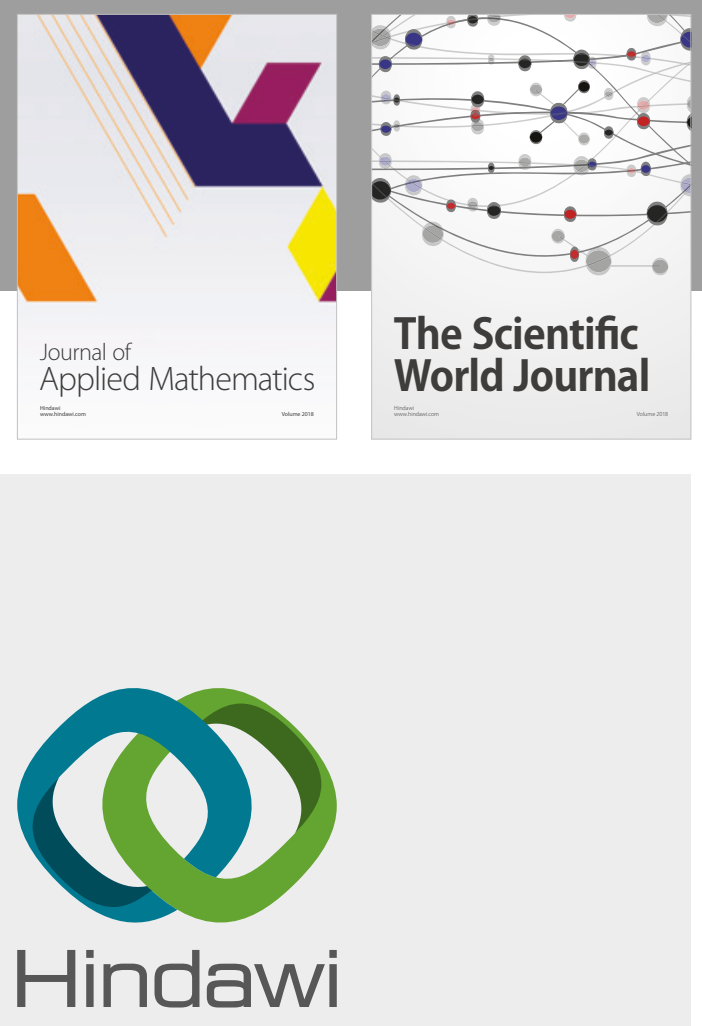

Submit your manuscripts at

www.hindawi.com

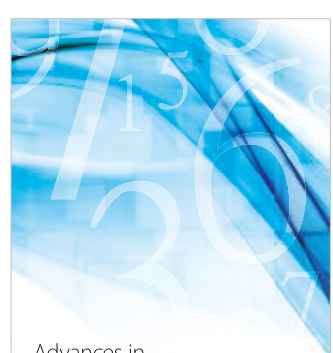

Advances in
Numerical Analysis
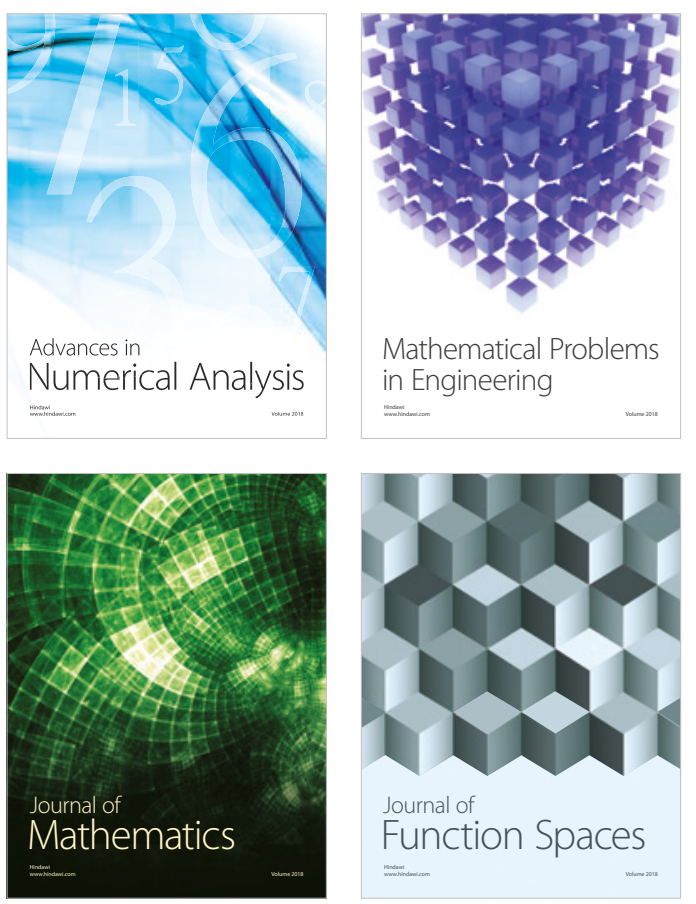

Mathematical Problems in Engineering

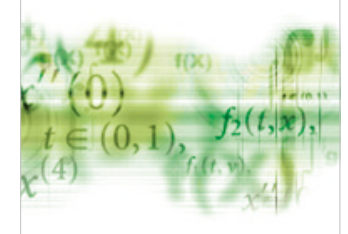

International Journal of

Differential Equations

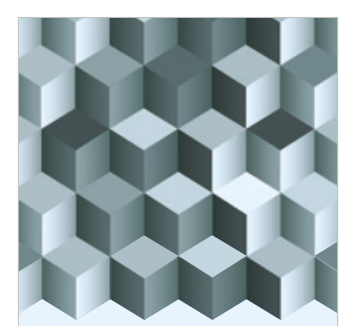

Journal of

Function Spaces

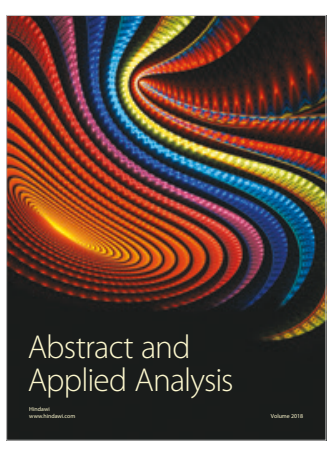

The Scientific

World Journal

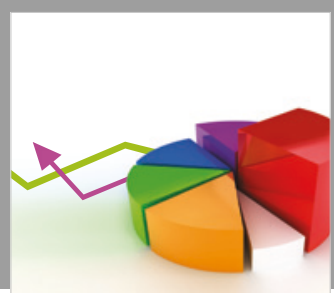

Journal of

Probability and Statistics
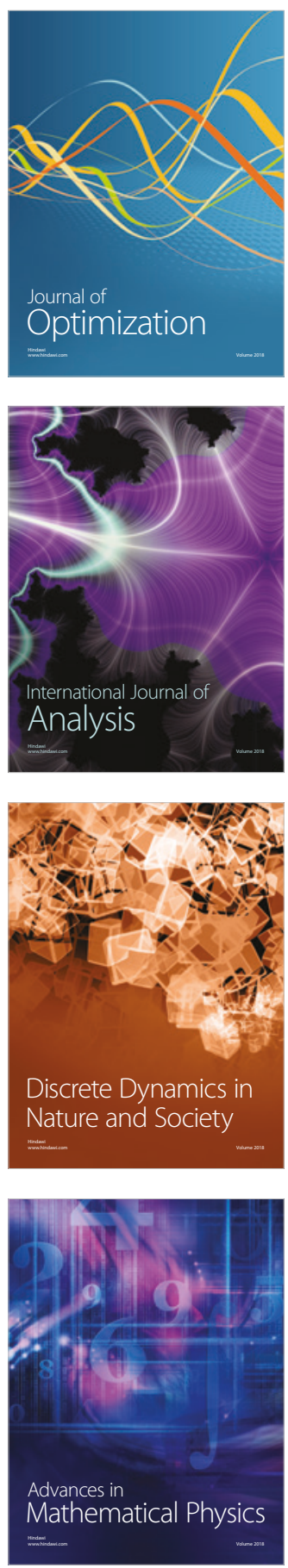